\title{
Régis Debray du médiateur au médiologue
}

Nicole Denoit

\section{OpenEdition}

\section{Journals}

Édition électronique

URL : http://journals.openedition.org/communicationorganisation/1954

DOI : 10.4000/communicationorganisation. 1954

ISSN : $1775-3546$

Éditeur

Presses universitaires de Bordeaux

Édition imprimée

Date de publication : 1 mai 1997

ISSN : 1168-5549

\section{Référence électronique}

"Régis Debray du médiateur au médiologue », Communication et organisation [En ligne], 11 | 1997, mis en ligne le 26 mars 2012, consulté le 02 mai 2019. URL : http://journals.openedition.org/ communicationorganisation/1954 ; DOI : 10.4000/communicationorganisation.1954

Ce document a été généré automatiquement le 2 mai 2019.

(C) Presses universitaires de Bordeaux 


\title{
Régis Debray du médiateur au médiologue
}

\author{
Nicole Denoit
}

\section{NOTE DE L'ÉDITEUR}

Propos recueillis par Nicole Denoit

1 Il n'est pas utile de présenter Régis Debray, que nous remercions d'avoir accordé une entrevue à Communication \& Organisation. Nicole Denoit l'a rencontré pour nous en Mai dernier et l'entretien a porté essentiellement sur son dernier ouvrage Transmettre publié en février 1997 aux éditions Odile Jacob.

2 Question: Vous venez de publier un nouvel ouvrage médiologique intitulé Transmettre. Ce mot pour le médiologue que vous êtes se révèle une arme sémantique très efficace pour affronter, vous le rappelez vous-même, "le système des forces rivales qui tendent à éliminer ou à annexer la médiologie ». Vous semblez préférer aux sociétés de communication une société de transmission. J'aimerais que vous précisiez pourquoi, ce qui serait une manière de reprendre quelques idées importantes de votre ouvrage.

D'abord ce n'est pas une trouvaille stratégique ni une ruse politique. On ne se détermine pas à l'intérieur d'un jeu d'échec, avec un échiquier, par rapport à de supposés adversaires. Simplement, j'ai toujours été mal à l'aise dans la problématique de la communication parce qu'elle me semble courte philosophiquement et courte historiquement. Ce qui m'intéresse dans la transmission c'est l'historicité du problème. La communication est pour moi l'expression d'une société fondée sur l'espace et non sur le temps, d'une société qui oublie sa propre temporalité, qui oublie sa profondeur de temps. C'est pour moi une sorte d'hypostase technologique d'un rapport duel entre un émetteur et un récepteur, c'est du téléphone : «Allô ? Y a-t-il quelqu'un? Allô - tu es là ?»

4 En fait ce qui intéresse le propos médiologique, c'est le devenir force des idées, l'efficacité symbolique, c'est-à-dire une métamorphose historique. La transmission c'est la transformation d'une parole en événement, d'une doctrine en école, d'une école de 
pensée en partie... Bref, la dimension diachronique est fondamentale et c'est cette dimension que la communication ne donne pas. Donc, la transmission nous tourne d'emblée vers une anthropologie culturelle et peut-être une anthropologie tout court, ou plutôt une anthropologie qui retrouverait l'histoire, alors que l'anthropologie s'en écartait quand elle était structurale. Donc, cette notion de transmission a l'avantage d'être à la fois plus globale et plus opérationnelle que celle de communication. Cela dit, il n'y a évidemment pas de transmission sans acte de communication. Les actes de communication trouvent, me semble-t-il, leur explication et leur logique dans la transmission à laquelle ils participent. Évidemment, la problématique de la transmission exclut l'intersubjectif ou l'interpersonnel parce que cela est vrai qu'un rapport de maître à disciple est un rapport personnel toujours pris dans une instruction ou un devenir institutionnel. Ce sont des rapports d'autorité qui ne sont pas de type charismatique improvisé.

:Et qui sortent peut-être d'un égoïsme, d'un narcissisme de la communication c'est-à-dire que, même si cet aspect-là persiste, il est pris en charge par quelque chose qui est collectif, qui est peut-être aussi moral. Je me demandais si, avec la transmission, on ne touchait pas à un problème de morale alors qu'on peut l'évacuer dans le problème de communication.

Commençons par rappeler que nous entendons par communication le transport d'informations dans l'espace et par transmission le transport d'informations dans le temps. C'est une ventilation un peu sommaire et brutale mais qui peut éclaircir nos idées dans ce domaine. Mais il est vrai qu'il y a un enjeu moral dans la transmission qui est tout simplement l'existence de l'humanité en tant que culture : "L'humanité comme un seul peuple", selon le mot d'Auguste Comte, ou simplement la nation comme une seule histoire, enfin l'être humain comme être historique, contrairement à l'animal, c'est pourquoi je dis : les animaux communiquent, ils ne transmettent pas.

Question : Les animaux semblent pourtant transmettre une éducation à leurs petits.

8 C'est très important. On le dit effectivement de certains primates, les chimpanzés. Pour moi cette éducation consiste à développer un programme génétique très ample dans le petit. Et par ailleurs, je dis qu'il n'y a pas de transmission car la transmission est un cumul génératif, c'est-à-dire une continuité inventive. Les chats s'éduquent les uns les autres, de géniteurs à petits. Ils ne se transmettent rien, c'est-à-dire qu'ils répètent à chaque fois le même processus.

Question : Je ne suis pas convaincue de cela

10 Vous ne croyez pas qu'une mère chatte transmet à son petit les mêmes comportements, les mêmes réflexes que le petit chat transmettra...

11 Question: Pourtant on observe des différences énormes. Déjà, la même chatte n'éduque pas ses petits de la même manière. Elle les individualise. Elle en choisit certains qu'elle forme avec beaucoup d'insistance. Par ailleurs, on voit des chattes qui n'éduquent pas ou très peu leur petits et pourtant le potentiel génétique est là. Je reste réservée sur ce sujet.

12 C'est très important et je vous écoute avec beaucoup d'intérêt. Je serai instruit, c'est le moins qu'on puisse dire, je serai, pas bouleversé mais remué, par un démenti que j'attends volontiers.

13 C'est en éthologie qu'on va faire les plus grands progrès. Je suis persuadé que l'éthologie sera au prochain siècle une science maîtresse. Je suis très faible en éthologie. Je crois que l'anthropologie sera un sous-produit de l'éthologie et que les animaux vont apprendre à l'homme ce qu'est l'homme et, comme on connaît encore assez peu de choses sur les 
animaux, je suis très prudent. De plus, je ne suis pas du tout un spécialiste. Ce que je disais concerne ce que j'ai pu lire sur les abeilles, les dauphins, tous ces transmetteurs de signes à distance, dans l'espace.

Question: C'est peut-être justement sur ce point qu'il y a quelque chose d'un peu sommaire à ce propos car nous évoquions précédemment un rapport de grande proximité et c'est peut-être là, dans cette proximité, qu'il y aurait un phénomène de transmission qui s'ajouterait à un processus de communication. Mais je ne suis pas non plus une spécialiste...

Mais c'est très intéressant. Il faudrait consulter les primatologues. J'en ai entendu un dans un séminaire sur Leroi-Gourhan qui défendait l'idée que les chimpanzés se transmettent de génération en génération des comportements mais ce n'était pas très convaincant. Pour moi cela reste de l'ordre de l'actualisation d'un potentiel génératif présent dans le legs génétique de l'animal, dans ses synapses, dans son petit bagage neurologique.

Vous n'allez pas me contester que les chats d'aujourd'hui sont identiques aux chats des égyptiens?

Question: Qu'est-ce qui vous permet de le dire?

Ont-ils appris quelque chose de nouveau? Question: Avons-nous appris quelque chose de nouveau?

On a l'automobile, le vaccin antipoliomyélite, on a des maisons, on a du macadam, enfin bref, on a l'humanité.

Question: Pour ce qui est de la survie, pour ce qui est des fonctions vitales, finalement la similitude est très grande.

Qu'il y ait un socle biologique inaltérable, j'en conviens, mais le développement scientifique et technique, la prise de possession de l'espace...

Question: Oui, je ne vais pas dire à un médiologue que rien n'a changé. Cela serait ne rien avoir compris entre autres à la médiologie!

Vous touchez un point très sensible qui est le point crucial, névralgique, sur lequel je dois avouer que je suis très perplexe moi-même, c'est pourquoi vos questions me touchent. Je ne sais pas bien articuler ce qui change et ce qui ne change pas. Je sais qu'il y a des choses qui ne changent pas dans l'homme et je suis le premier à le dire. Je ne pense pas que l'inconscient individuel ait une histoire, je ne pense pas que l'inconscient collectif ait une histoire. Je pense que les sentiments et les conduites de domination sont à peu près les mêmes. Je crois dans la stabilité d'une nature humaine.

Par ailleurs je suis bien forcé de voir que la technique renverse les dogmes ou plutôt rebat les cartes du jeu anthropologique à chaque fois, à chaque remplacement d'un macrosystème technique par un autre.

La question est de savoir si on pense de la même manière avant l'invention de l'écriture et après, avant l'invention de l'imprimerie et après, si l'invention des écritures électroniques vont changer le fonctionnement de la pensée. Voilà une question importante. J'ai envie de répondre oui et non.

Question: Parce que vous devez être comme moi fasciné par l'adéquation que l'on trouve à la lecture des textes très anciens.

On circule dans la pensée ancienne. On circule dans Platon et dans Saint Thomas.

Question: Dans tous vos ouvrages vous avez de belles formules qui résonnent efficacement parce que déjà du point de vue sonore vous êtes médiologue, c'est à dire que vous rapprochez des réalités: 
"mémoire ethnique, tendance technique "ou encore "les réactivations des orthodoxies par les ordinateurs ". Dans une sorte de prose poétique, ces effets sonores jouent un rôle de médiation entre des réalités opposables.

C'est-à-dire on invente des ritournelles, des euphonies qui facilitent la mise en mémoire. C'est une technique de transmission. Elle est immémoriale.

Question : Elle fonctionne très bien chez vous. De plus, les effets sonores, les trouvailles linguistiques soulignent l'originalité de votre démarche. Ainsi la médiologie vous conduit à observer " une chaîne de tremblements d'états » et en ce sens " la médiologie confinerait à une sismologie ». Pouvez-vous développer quelques éléments de cette comparaison?

La comparaison est avec la géologie. On suppose que les sociétés sont composées de diverses strates, qu'il y a en dessous, à la base, des plaques de culture composées de sédiments religieux, immémoriaux, de l'empreinte d'un territoire sur les mentalités. Sur cette plaque, relativement immobile ou à évolution lente de la culture, s'installe une croûte technologique, c'est-à-dire des acquisitions beaucoup plus récentes beaucoup plus fragiles qui touchent le domaine des ustensiles qui est donc moins incorporée profondément que les valeurs ou les enseignements de l'enfance liés à une religion, une structure familiale...

33 Il y a donc une croûte technologique qui avance très vite et une plaque culturelle qui est stable. Il y a une sorte de ligne de faille entre ces deux couches géologiques qui provoque tous ces tremblements intégristes que l'on voit aujourd'hui. Ce n'est qu'une métaphore. Je ne conceptualise pas les phénomènes, je les décris d'une autre façon. Simplement je crois que nous vivons cette contradiction dans le Tiers-Monde mais aussi en France même, et on pourrait lire l'actualité avec cette grille, aussi bien le F.I.S. algérien, le Front National français. On peut lire cette actualité avec cette grille d'interprétation comme étant la résistance des socles culturels aux attaques du bouleversement technologique.

Question: "Notre époque n'est plus, dites-vous, aux artistes ingénieurs de la Renaissance. Aujourd'hui, à chacun sa spécialité et nous avons donc besoin d'entremetteurs ». Or, je le rappelle, le thème du prochain numéro de notre revue est " la médiation ", le médiateur, ce qui n'est pas tout à fait pareil que le médiologue. Le médiateur serait celui qui met en relation, harmonise, tente une conciliation dans la pratique entre deux éléments qui s'ignorent parfois dangereusement ou s'affrontent et lorsqu'ils ne s'ignorent plus c'est souvent pour s'affronter. Il se pourrait que le médiateur et le médiologue soient de la même race. Leurs identités sont-elles cependant superposables? Comment définir aujourd'hui celle du médiologue par rapport à celle du médiateur?

Le médiologue est celui qui réfléchit sur les médiateurs. Ce peut-être un médiateur qui réfléchit sur lui-même et, au fond, la médiologie est peut-être née comme cela. Un monsieur qui essaye de faire du journalisme politique ou qui essaye de faire changer les choses en prenant l'arme des mots se dit: «mais comment les mots deviennent-ils des choses ou font bouger les choses? Quelles sont les médiations entre un état de pensée et un état de fait, un état de société ?» Donc, un médiateur professionnel réfléchit sur les conditions d'exercice de sa fonction médiatrice et la médiologie peut naitre ainsi. Je dirais que le médiologue s'intéresse évidemment aux métiers de la médiation, aux travers, aux vices, aux perversions de cette fonction sociale de médiation qui sont aujourd'hui mises en évidence par les technologies de transmission qui donnent le premier rôle à l'interprète, la primauté de l'interprète sur le compositeur, de l'acteur sur le metteur en scène, ou du metteur en scène de théâtre sur l'auteur de théâtre, ou du conservateur de 
musée, du commissaire d'exposition sur le peintre qu'il expose. Ce sont tous les métiers de la médiation, de la mise en scène culturelle.

Lorsque la médiation se prend pour le propre fin, là nous sommes dans une perversion.

Question: Vous soulignez le décalage entre l'innovation et la mémoire, entre l'état d'une culture, d'une sensibilité, et les propositions techniques qui lui sont faites. Peut-on dire que le médiateur serait celui qui, dans l'entre-deux tente de maintenir à la bonne distance les plaques de l'innovation et celle de la mémoire, le réel et notre rêve de réalité, la réalité et notre désir?

En ce sens le médiateur se séparait du médiologue dont le rôle ne vise pas à faire coexister, à rendre possible les choses, à les concilier? Pensez-vous être un conciliateur? Les réactions que vous suscitez tendraient à prouver le contraire.

Je ne sais pas trop si je peux vous suivre dans ces jeux de mots amusants.

Question: Je me demande pourquoi vous êtes si peu conciliateur dans le moment présent. Je ne parle pas de ce qui a pu se passer autrefois.

Vous savez, une discipline nouvelle, elle commence par abattre des murs. Tout ce qui s'est fait depuis des siècles d'intéressant, ça a consisté à mettre en relation des domaines qui n'étaient pas en relation. C'est ce que les sociologues ont fait : ils ont abattu le mur entre l'individuel et le collectif, c'est ce que les écologues ont fait: ils ont abattu le mur entre l'inerte et le vivant. Que fait un médiologue? Il abat le mur de séparation entre le symbolique d'un côté, le technique de l'autre. Donc, par définition, ce n'est pas un conciliateur, c'est un passeur, c'est quelqu'un qui fait rentrer un domaine dans l'autre et vice versa. C'est toujours très embêtant pour chacun des domaines mis en relation parce qu'on voit cet intrus faire des choses pas convenables.

Question: Et puis qui ne fait que penser, ce qui est désagréable. On voudrait le saisir un peu...

Voilà. Exactement. Il y a toutes sortes de proposition d'annexion de la médiologie, par les sociologues, les historiens. Ils ont peut-être raison, je ne sais pas, mais en tout cas je dirai que le médiologue est celui qui a vraiment pour fonction de mettre autour d'une table des gens qui ne se parlent pas.

Question: En tout cas, ce qui semble assez évident, est que vous souhaitez être davantage un transmetteur de culture qu'un communicateur. Et finalement il semblerait que vous soyez plus préoccupé de transmettre une culture que vous sentez menacée notamment un certain rapport à l'image que de communiquer vos convictions. Je me demande comment cela peut se faire l'un sans l'autre. Le talent avec lequel vous évoquez celte culture de l'image s'inscrit certes dans les mémoires durablement. C'est votre projet. D'abord vivifier cette culture en en parlant, en l'inscrivant dans les mémoires par des formules, des images. Ce sont des images sur des images, des mots qui font image. C'est par ce talent de transmetteur que vous souhaitez faire cuvre mais peuton transmettre sans communiquer?

Je ferai subir à votre question un petit déplacement pour y répondre.

Peut-on faire du savoir sans faire de la morale?

Peut-on faire de l'écologie au sens scientifique sans faire de l'écologie au sens politique?

Je dis que c'est beaucoup plus compliqué de faire du savoir que de faire de la morale. N'importe qui fait de la morale. Mais comprendre des corrélations, mettre à jour des systèmes de causes ou de covariations ou d'interdépendances me semble beaucoup plus important que la classique exhortation morale. 

Manche. vendre, vous trouverez preneur.

Question : Avec vous on ne sait jamais...

Question: Des inquiétudes, des angoisses.

Question : De l'immédiat.

Il est bien vrai que, lorsque vous vous occupez de l'équilibre des populations dans un écosystème vous êtes plus sensible qu'un autre à la réglementation du dégazage dans la

Si vous vous intéressez à l'évolution des colonies de pétrels du côté d'Etretat, ce qui se passe avec Torrey Canyon en 67 vous bouleverse plus.

Il ne faut pas confondre une cause sociale avec une cause scientifique, même si la cause scientifique vous rend plus sensible. C'est vrai que, quand un médiologue voit les photocopieuses marcher sans contrôle dans les bibliothèques, quand il voit monter la part publicitaire dans les chaînes de télévision, quand il voit les bicyclettes qui réapparaissent dans les rues, il est, tantôt mécontent, tantôt content, mais il est plus partie prenante. Ceci dit je n'entends pas faire de l'idéologie. Je souhaite comprendre parce que j'ai une formation philosophique et rationaliste. Je ne suis pas prophète. C'est d'ailleurs ennuyeux parce qu'il y a une demande de prophétie, d'apocalypse. Si vous avez une apocalypse à

Avec moi, non. Il n'y a pas d'apocalypse. Il y a des trucs bizarres, ambigus...

Des inquiétudes, des angoisses, non. Des surprises... mais je pense que tout renaît.

Question: Quelque chose me préoccupe dans vos propos que je ne suis pas certaine d'avoir compris. Vous accordez une attention particulière à l'aspect matériel de la transmission. Vous insistez sur le fait que la transmission, à la différence de la communication, se dit des biens comme des idées et pourtant, cet intérêt n'a pas d'incidence sur votre méfiance à l'égard de la "ligne chair » qui est le versant à mon avis concret, matériel de la communication et vous lui préférez la "ligne verbe", dans vos propos antérieurs. Non pas dans Transmettre. Dans le Cours de médiologie générale, vous manifestez une nostalgie de la « ligne verbe » et vous êtes inquiet par rapport à la «ligne chair ». Or vous êtes sensible au fait que, dans la transmission, comme une valorisation supplémentaire par rapport à la communication, il y a une attention particulière au matériel. Je suis donc surprise de votre réticence à l'égard de la "ligne chair » qui s'impose dans notre société.

Vous avez raison d'être perturbée parce que je le serais aussi si c'était vrai mais le fondement, le noyau dur de la médiologie c'est l'incarnation. C'est « et le verbe s'est fait chair ». C'est le mystère médiologique premier. Donc, c'est le devenir chair du verbe qui intéresse le médiologue. En ce sens, je suis peut-être méfiant envers les dérivations de la « ligne chair », envers les vertiges et les excès de la « ligne chair » qui sont, en gros, la communication charismatique, l'hypnose, le culte du sensible, du sensoriel.

De l'immédiat. Oui. C'est vrai que nous sommes là dans l'extrême d'une «ligne chair ». C'est vrai que supprimer l'universel au bénéfice de l'individu... Ce nominalisme quasiment officiel de notre vie sociale fait que je suis effectivement sur la défensive mais, fondamentalement, je suis étranger à la «ligne verbe» qui serait, en gros, la ligne judaïque ou logicienne. Si le christianisme me fascine à ce point c'est qu'il a trouvé l'équilibre entre le verbe et la chair et je me sens tout à fait dans la filiation chrétienne.

Question: Vous déplorez dans la «ligne chair », cette immédiateté, le fait que la mémoire ne soit pas prise en compte. Je crois que les artistes contemporains, sont très sensibles aux traces mais à des traces qui ne font pas référence à une mémoire de quelque chose qui serait antérieur mais à la 
trace du moment présent qu'ils vont transmettre. Un peu comme s'il y avait une amnésie et qu'on recommence. Comme si, à partir de maintenant il y allait avoir une mémoire.

61 Ce n'est pas si simple. Cette "ligne chair» de l'immédiat, ce que vous n'aimez pas beaucoup, je crois, les happenings, les performances, je ne suis pas sûre que ce soit une «ligne chair » qui soit détachée de la mémoire, qui soit donc aussi frustre et aussi sensorielle, brutale. Je me demande s'il n'y a pas là quelque chose qui réconcilie quand même la mémoire et l'immédiateté. Cela serait la "mémoire de l'immédiateté"

62 C'est très joliment décrit par vous et je ne pense qu'approuver si c'est ainsi. Et de toutes façons, que j'approuve ou pas cela ne changera rien, mais je suis d'accord avec ce que vous dites.

63 J'ai eu le grand plaisir de recueillir ces propos devant une magnifique toile d'Ernest Pignon Ernest dont le travail s'inscrit très symboliquement dans un rapport au temps : concevoir des œuvres sur des supports fragiles, éphémères, qui resteront placés -livrés aux assauts des hommes peut-être, des intempéries certainement - sur les lieux mêmes qui ont inspiré leur création. Des lieux de mémoire, non pas nécessairement prestigieux mais d'une mémoire populaire, collective, quotidienne, celle de la rue, celle des habitants les plus modestes des différents quartiers de Naples. On a pu remarquer depuis, comme cette vulnérabilité liée à la nature même de l'œuvre d'art, a révélé chez les napolitains un respect, une volonté de protéger l'éphémère. 